\title{
Impact of neoadjuvant intensity-modulated radiation therapy on borderline resectable pancreatic cancer with arterial abutment; a prospective, open-label, phase II study in a single institution
}

Toshihiko Masui ${ }^{1 *}$, Kazuyuki Nagai ${ }^{1}$, Takayuki Anazawa ${ }^{1}$, Asahi Sato ${ }^{1}$, Yuichiro Uchida ${ }^{1}$, Kenzo Nakano ${ }^{1}$, Akitada Yogo', Akihiro Kaneda', Naoto Nakamura', Michio Yoshimura², Takashi Mizowaki², Norimitsu Uza ${ }^{3}$, Akihisa Fukuda ${ }^{3}$, Shigemi Matsumoto ${ }^{4}$, Masashi Kanai ${ }^{5}$, Hiroyoshi Isoda ${ }^{6}$, Masaki Mizumoto ${ }^{1}$, Satoru Seo ${ }^{1}$, Koichiro Hata ${ }^{1}$, Kojiro Taura ${ }^{1}$, Yoshiya Kawaguchi ${ }^{1}$, Kyoichi Takaori ${ }^{1}$, Shinji Uemoto ${ }^{1}$ and Etsuro Hatano ${ }^{1}$

\begin{abstract}
Background: Borderline resectable pancreatic cancer (BRPC) is a category of pancreatic cancer that is anatomically widely spread, and curative resection is uncommon with upfront surgery. Intensity-modulated radiation therapy (IMRT) is a form of radiation therapy that delivers precise radiation to a tumor while minimizing the dose to surrounding normal tissues. Here, we conducted a phase 2 study to estimate the curability and efficacy of neoadjuvant chemoradiotherapy using IMRT (NACIMRT) for patients with BRPC with arterial abutment (BRPC-A).

Methods: A total of 49 BRPC-A patients were enrolled in this study and were treated at our hospital according to the study protocol between June 2013 and March 2021. The primary endpoint was microscopically margin-negative resection (RO) rates and we subsequently analyzed safety, histological effect of the treatment as well as survivals among patients with NACIMRT.
\end{abstract}

Results: Twenty-nine patients (59.2\%) received pancreatectomy after NACIMRT. The R0 rate in resection patients was 93.1\% and that in the whole cohort was $55.1 \%$. No mortality was encountered. Local therapeutic effects as assessed by Evans classification showed good therapeutic effect (Grade 1, 3.4\%; Grade 2a, 31.0\%; Grade 2b, 48.3\%; Grade 3, 3.4\%; Grade 4, 3.4\%). Median disease-free survival was 15.5 months. Median overall survival in the whole cohort was 35.1 months. The only independent prognostic pre-NACIMRT factor identified was serum carbohydrate antigen 19-9 (CA19-9) > $400 \mathrm{U} / \mathrm{ml}$ before NACIMRT.

Conclusions: NACIMRT showed preferable outcome without significant operative morbidity for BRPC-A patients. NACIMRT contributes to good local tumor control, but a high initial serum CA19-9 implies poor prognosis even after neoadjuvant treatment.

\footnotetext{
*Correspondence: tmasui@kuhp.kyoto-u.ac.jp

1 Department of Surgery, Graduate School of Medicine, Kyoto University,

Kyoto, Japan

Full list of author information is available at the end of the article
}

(C) The Author(s) 2022. Open Access This article is licensed under a Creative Commons Attribution 4.0 International License, which permits use, sharing, adaptation, distribution and reproduction in any medium or format, as long as you give appropriate credit to the original author(s) and the source, provide a link to the Creative Commons licence, and indicate if changes were made. The images or other third party material in this article are included in the article's Creative Commons licence, unless indicated otherwise in a credit line to the material. If material is not included in the article's Creative Commons licence and your intended use is not permitted by statutory regulation or exceeds the permitted use, you will need to obtain permission directly from the copyright holder. To view a copy of this licence, visit http://creativecommons.org/licenses/by/4.0/. The Creative Commons Public Domain Dedication waiver (http://creativeco mmons.org/publicdomain/zero/1.0/) applies to the data made available in this article, unless otherwise stated in a credit line to the data. 
Trial Registration: UMIN-CTR Clinical Trial: https://upload.umin.ac.jp/cgi-open-bin/ctr_e/ctr_view.cgi?recptno= R000011776

Registration number: UMIN000010113.

Date of first registration: 01/03/2013,

Keywords: Neoadjuvant therapy, Pancreatic cancer, Intensity-Modulated Radiotherapy, Surgery

\section{Background}

Pancreatic cancer is one of the most poorly prognosed malignancies, with high mortality rates worldwide [1]. This disease is the fourth leading cause of cancer deaths in Japan, and its incidence is rising with the aging of the population [2]. For pancreatic cancer without metastasis, surgical resection offers the highest cure rate. However, curative resection is sometimes difficult if the tumor is overly close to vital arteries or veins. The National Comprehensive Cancer Network (NCCN) has proposed the category of "borderline resectable pancreatic cancer" (BRPC) for such tumors [3]. BRPC is defined as a tumor meeting any of the following criteria: BRPC-A; 1) focal tumor abutment (in contact with $\leq 180^{\circ}$ of vessel circumference) of the superior mesenteric artery (SMA) or of the celiac axis (CA); 2) encasement of common hepatic artery (CHA) but not to the CA or proper hepatic artery (PHA); or BRPC-V; 3) involvement of the superior mesenteric vein (SMV)/ portal vein (PV) with abutment more than $180^{\circ}$. Given these definitions, BRPC-A represents a particularly difficult entity when trying to achieve curative resection [4]. Recently, neoadjuvant therapy with FOLFIRINOX [5] or gemcitabine plus radiotherapy [6, 7] for BRPC patients has shown favorable microscopically margin-negative resection (R0) rates, but the contributions to survival have remained contentious. Similarly, our previous phase 2 study with gemcitabine and S-1 showed better R0 rates compared to upfront surgery, but failed to show any survival advantage for those patients [8]. Because of the high rates of R0 after neoadjuvant therapy, patients with BRPC-A might benefit most from neoadjuvant therapy with additional radiation.

Intensity-modulated radiation therapy (IMRT) is a radiotherapeutic technique that allows higher radiation doses to be focused to regions while minimizing the dose to normal tissue. The advantage of IMRT over conventional radiation therapy is that it maximizes the effect on the target tissue and reduces the toxicity to the surrounding normal tissue. $[9,10]$. Although IMRT has been used for other tumors such as prostate cancer [11, 12] and nasopharyngeal carcinoma [13, 14], few data has been accumulated on its efficacy in treating patients with pancreatic cancer, and even less on survival outcomes [15, 16], because the target tissue shifts with respiration, making it difficult to irradiate the tissue accurately [17]. We have reported a favorable outcome of IMRT to patients with non-metastatic locally advanced pancreatic cancer [18].

Here, we have conducted a prospective phase 2 study for BRPC-A patients to analyze the impact of neoadjuvant chemoradiotherapy using IMRT (NACIMRT) with gemcitabine on surgical curability and survival.

\section{Methods}

\section{Study design and Patients}

This study was conducted as a prospective phase II study of neoadjuvant treatment with IMRT plus gemcitabine (UMIN000010113) for BRPC-A patients. The primary endpoint was the R0 rate to evaluate the effect of IMRT (total dose, 42 Gy) with gemcitabine as neoadjuvant therapy for BRPC-A. All patients with pancreatic tumors classified as BRPC-A according to NCCN 2009 guidelines diagnosed at our hospital between June 2013 and March 2021 and who provided consent were enrolled to this study. The extent of tumor involvement as BRPC-A was assessed from multidetector-row computed tomography (MDCT) using a multiphase contrast-enhanced technique and evaluated by a multidisciplinary team for pancreatic cancer comprising doctors from the Department of Surgery, Department of Gastroenterology, Department of Radiation Oncology, Department of Clinical Oncology and Department of Diagnostic Imaging.

Inclusion criteria for BRPC-A pancreatic cancer in this study were as follows. In brief, with the contrastenhanced MDCT, patients showing tumor abutment with the SMA at $=<180$ degree of the vessel circumference, or tumor abutment with the CHA allowing complete resection were defined as BRPC-A. Tumors with abutment of the CA but not to the aorta that could be completely resected by distal pancreatectomy with celiac axis resection were also categorized as BRPC-A. Other inclusion criteria were as follows: histologically confirmed pancreatic ductal adenocarcinoma; age $>20$ but $<80$ years; and ECOG performance status of 0 or 1 , no distant metastasis in the thorax, abdomen or pelvis on dynamic contrast-enhanced MDCT, on positron emission tomography with 2-deoxy-2-[ $\left[{ }^{18} \mathrm{~F}\right]$ fluoro-D-glucose (FDG-PET) and on magnetic resonance imaging (MRI) with contrast medium of gadolinium-ethoxybenzyl diethylenetriaminepentaacetic acid (EOB-MRI), no pre-treatment for 
current pancreatic cancer, and no hematological dysfunction or that of the main organs.

Exclusion criteria were as follows: interstitial pneumonitis; history of irradiation to the upper abdomen; serious comorbidities (heart failure, renal failure, liver failure, bleeding peptic ulcer, intestinal paralysis, intestinal obstruction, uncontrolled diabetes); moderate or severe ascites or pleural effusion; history of active cancer (concurrent multiple cancers or heterogeneous multiple cancers with a disease-free interval of less than 3 years); or expectant or nursing women. The Ethics Committee of Kyoto University approved this study, and each patient gave informed consent prior to participation.

\section{Neoadjuvant chemoradiotherapy}

The treatment for NACIMRT is presented in Fig. 1. After the tumor was diagnosed histologically as adenocarcinoma by endoscopic ultrasonographic fine needle aspiration, patients were initially administered gemcitabine 3 times (days 1,8 , and 22) at a dose of $1000 \mathrm{mg} / \mathrm{m}^{2}$ before chemoradiotherapy. On starting radiotherapy, gemcitabine was administered at a dose of $1000 \mathrm{mg} / \mathrm{m}^{2}$ (days 1,8 , and 22) concurrent with IMRT. When grade 4 or worse neutrocytopenia or thrombocytopenia occurred, chemotherapy was stopped for a week. For IMRT planning, gross tumor volume (GTV) included the pancreatic tumor and any lymph nodes $>1 \mathrm{~cm}$ in diameter. Clinical target volume (CTV) included the celiac and para-aortic lymph node basins, in addition to the GTV plus a 5-mm margin, according to our institutional contouring guidelines. Organs at risk were the liver, stomach, duodenum, small intestine, colon and kidneys, as well as the spinal cord, and were delineated on expiratory-phase CT. The planning target volume (PTV) was defined as the CTV with a 5-mm margin in all directions. The prescription dose of 42 Gy administered in 15 fractions was specified as D95 (the dose covering $95 \%$ of the target structure) to PTV-boost. PTV-boost is a volume that subtracted the stomach plus $10-\mathrm{mm}$, and the duodenum plus $5-\mathrm{mm}$ margins from the PTV. IMRT was used to generate optimized treatment plans for each patient. Breath-hold method was adopted for the management of tumor respiratory motion and daily cone beam CT before each treatment was used to determine the daily set-up errors. Radiation treatment was delivered with volumetric modulated arc therapy techniques.

\section{Resection and adjuvant chemotherapy}

Patients were evaluated for resection within 4 weeks after neoadjuvant therapy using MDCT, EOB-MRI, and FDG-PET and were examined by our multidisciplinary pancreatic cancer treatment team. In the absence of clear technical unresectability, resection was attempted between 4 and 8 weeks after finishing neoadjuvant

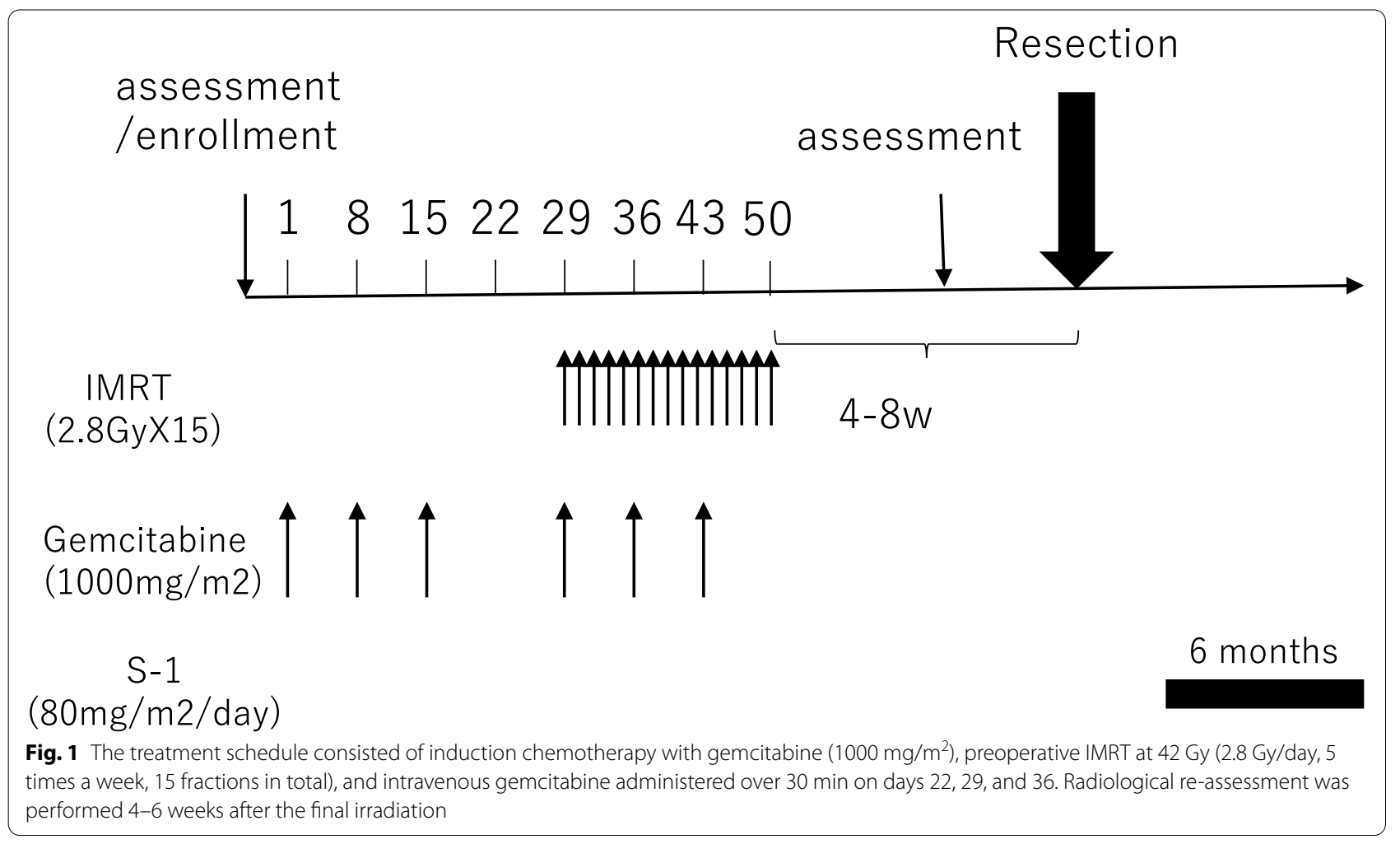


radiotherapy. Pancreaticoduodenectomy, distal or total pancreatectomy (and resection of any involved tissues) was performed according to the tumor location. Operative findings, surgical complications, and histopathology were recorded. S-1 at a dose of $80 \mathrm{mg} / \mathrm{m}^{2} /$ day was administered on days 1-28 of a 42-day cycle for 6 months as adjuvant chemotherapy, starting 4-8 weeks after resection.

\section{Assessment}

Resection margins were determined as positive (R1) if malignant cells were observed at the surface of the resected specimen (0-mm margin rule), the plexus around the SMA or CHA, duodenum, bile duct, or retroperitoneal tissue. If vein was concomitantly resected, the vein margin was examined additionally.

Follow-up data were examined on medical records up to August 2021. Patients' status was evaluated by contrast-enhanced CT every 3 months for the first 2 years, then every 6 months thereafter. The first site of disease recurrence was defined as follows: A new low-density mass in the peripancreatic and mesenteric root area was considered a locoregional recurrence. For locoregional failure-free interval (LFFI) analysis, locoregional failure was only the event of interest and was defined as the appearance of tumors in the region of the resected pancreatic bed and root of the mesentery. For distant metastasis-free interval (DMFI) analysis, distant metastatic failure was the event of interest and was defined as a new low-density region in the liver or lungs as well as new ascites on ultrasonography or $\mathrm{CT}$, subsequently confirmed by cytology as peritoneal dissemination. Disease-free survival (DFS) was calculated as the time from the date of surgery to that of initial recurrence. Overall survival (OS) was calculated as the time from the date of initial treatment to that of death. Tumor length was estimated based on the contrast-enhanced CT image before treatment and on the resected specimen. Toxicity events were recorded using the Common Terminology Criteria for Adverse Events (CTCAE version 4.0; https://ctep. cancer.gov/protocoldevelopment/electronic_applicatio ns/docs/CTCAE_4.03.xlsx). From the start of radiotherapy until two weeks after the end of chemoradiotherapy, weekly complete blood count and liver function tests were performed. Serum carbohydrate antigen 19-9 (CA19-9) concentration before treatment was evaluated after biliary drainage.

\section{Statistics}

We assumed that the R0 resection rate for BRPC-A patients after neoadjuvant therapy would be $10 \%$ to $30 \%$. Our null hypothesis was that the R0 resection rate for those BRPC-A patients confirmed by a radiology would be $<10 \%$. In the current trial, the proposed sample size was 40 patients, which was calculated according to the expected R0 resection rate of 30\%, a threshold of $10 \%$ and an alpha error of 0.05 , with a beta error of 0.05 . We expected that $90 \%$ of the enrolled patients would start NACIMRT as appropriate, so we decided that the actual sample size should be 45 patients. The final dataset was carefully assessed for clerical errors by three physicians (T.M., K.N., and T.A.). The primary endpoint and the secondary endpoints of the response rate, pathological response, R0 resection rate, surgical morbidity rate, acute and late toxicity of chemoradiation, DFS and OS were evaluated 6 months after the completion of enrollment. Data for continuous variables are expressed as median and range. Kaplan-Meier curves were created to estimate OS, and comparisons between groups were estimated using log-rank tests. To identify risk factors independently associated with survivals, multivariate Cox proportional hazards regression analysis was used. Values of $\mathrm{p}<0.05$ were considered significant. All statistical analyses were performed with JMP version 15.0 software (SAS Institute, Cary, NC).

\section{Results}

\section{Patient characteristics}

In total, 49 patients were enrolled between June 14, 2013 and March 16, 2021. Baseline characteristics of the cohort are summarized in Table 1. Thirty-four patients showed tumor involvement of the SMA (34/49, 69.3\%), while 9 patients showed involvement of the CHA. Thirtytwo patients $(32 / 49,65.3 \%)$ had tumors located in the head of the pancreas. PV occlusion or deformation was observed in 28 patients $(28 / 49,57.1 \%)$. Median tumor size was $25.7 \mathrm{~mm}$ and median carcinoembryonic antigen (CEA) was within the normal range $(<5.0 \mathrm{U} / \mathrm{ml})$, while median CA19-9 level was $111.4 \mathrm{U} / \mathrm{ml}$. Median maximum standardized uptake value (SUVmax) from FDG-PET was 6.4 and median neutrophil-to-lymphocyte ratio (NL ratio) before neoadjuvant treatment was 2.55 .

In the 30 patients who had biliary obstruction, biliary drainage was performed by biliary stenting with a metallic stent in 28 patients (93\%) and plastic stent in 2 patients (7\%),

\section{Safety and Clinical outcomes of NACIMRT}

The study diagram is shown in Fig. 2. The median time from the staging MDCT to the start of the neoadjuvant induction chemotherapy with gemcitabine was 13 days (range, 2-26). Of the 49 patients for whom IMRT was initiated, 47 patients completed IMRT with gemcitabine $(95.9 \%, 47 / 49)$. Two patients dropped out due to severe bone marrow suppression and an allergic reaction to gemcitabine. Of these, one patient underwent upfront 
Table 1 Patient Characteristics before NAC

\begin{tabular}{ll}
\hline Pre NACIMRT (at enrollment) & $n=49$ \\
age (years) & $68.6(46.0-77.8)$ \\
gender (male/female) & $29 / 20$ \\
head/body-tail & $32 / 17$ \\
radiographic arterial involvement & \\
celiac artery & 4 \\
common hepatic artery & 9 \\
replaced RHA & 2 \\
superior mesenteric artery & 34 \\
BRPV/without BRPV & $28 / 21$ \\
radiological tumor size (mm) & $25.7(15.0-47.0)$ \\
CEA before NACIMRT (U/ml) & $3.0(0.5-114.9)$ \\
CA19-9 before NACIMRT (U/ml) & $111.4(0.6-2451)$ \\
NL ratio before NACIMRT & $2.55(1.0-10.0)$ \\
SUV max before NACIMRT & $6.4(2.2-15.8)$ \\
NAC incompletion due to adverse event & $2 / 49(4.1 \%)$ \\
Post NACIMRT (at re-assessment after NACIMRT) & $n=47$ \\
radiological tumor size after NACIMRT (mm) & $21.5(11.7-50.1)$ \\
CEA after NACIMRT (U/ml) & $3.0(0.7-57.6)$ \\
CA19-9 after NACIMRT (U/ml) & $36.7(0.6-2298)$ \\
NL ratio after NACIMRT & $3.04(0.81-11.86)$ \\
SUV max after NACIMRT & $4.35(1.9-10.4)$ \\
RECIST > PR & $13 / 47(27.6 \%)$ \\
G3/4 adverse event & $10 / 47(21.3 \%)$ \\
\hline Abbrvatons:NAC neodjuvat therpy,MRTIntesity Mod & \\
\hline &
\end{tabular}

Abbreviations: NAC neoadjuvant therapy, IMRT Intensity Modulated Radiation Therapy, RHA right hepatic artery, BRPV borderline resectable pancreatic cancer with portal vein involvement, CEA carcinoembryonic antigen, CA19-9 carbohydrate antigen 19-9, NL ratio, neutrophil to lymphocyte ratio, SUV max, maximum standard uptake value

surgery and the other completed IMRT with S1. The median relative dose intensity of Gemcitabine was 100\% and the median total radiation dose was $42 \mathrm{~Gy}$. The preoperative therapy was well-tolerated by all the patients. The frequency of grade 3/4 toxicity in the patients who were initiated on NACIMRT was $24.4 \%$ (12/49). The adverse events are listed in Table 2.

In the group that completed NACIMRT, two patients showed local progression and 8 patients had distant metastasis; these 10 patients did not undergo surgical resection. Median CA19-9 concentration decreased from $111.4 \mathrm{U} / \mathrm{ml}$ to $36.7 \mathrm{U} / \mathrm{ml}$ in patients after completion of NACIMRT. In contrast, NL ratio increased from 2.55 to 3.04 after NACIMRT. The objective radiological response rate was $20.3 \%$ and the median radiographic tumor size reduced from $25.7 \mathrm{~mm}$ to $21.5 \mathrm{~mm}$. However, radiographic detachment from the major artery after NACIMRT was observed in only 5 patients (10.6\%).

\section{Surgical outcomes and pathological effects}

The median interval from completion of IMRT to surgery was 36 days (range, 28-44 days). In the 37 patients who underwent surgery, 5 patients had positive washing cytology and 3 patients had positive distant lymph node metastasis in the para-aortic region at laparotomy, resulting in 29 patients (59.2\%) with pancreatectomy.

These 29 patients underwent pancreatectomy with curative intent, and R0 resection was achieved in 27 patients (93.1\%) (Table 3). The overall R0 resection rate in the whole cohort was 55.1\% (27/49). According to the Evans classification, which pathologically estimates therapeutic effect, complete destruction of the tumor was observed in 1 patient, and $>90 \%$ destruction was observed in 1 patient, while $<50 \%$ destruction was observed in 10 patients (34.4\%). Pathological lymph node metastasis was observed in 10 patients (34.4\%).

Postoperative grade $3 / 4$ adverse events were observed in 6 patients with 5 patients of clinically relevant postoperative pancreatic fistula (CR-POPF), but no re-operations or in-hospital deaths were encountered. Median duration of the in-hospital stay after resection was 26.5 days.

Median values of both Albumin (Alb) and Cholinesterase (ChE) at 1 month after surgery were within normal ranges in the 29 patients of the eligible cohort. In the 29 patients with $\mathrm{R} 0 / 1$ resection in the cohort, 27 patients (93.1\%) started postoperative adjuvant therapy with S1 within 6 weeks after resection. Median relative dose intensity of S1 doses was $75 \%$.

\section{Survivals}

Kaplan-Meyer plots for survivals are shown in Fig. 3. Median follow-up for the censored patients was 21.0 months. Median OS in the whole cohort (intention to treat) was 35.1 months. (Fig. 3a) Patients with CA19-9>400 U/ml at enrollment showed significantly worse survival in this cohort (Fig. 3b). Median DFS of the eligible cohort was 15.5 months and median metastasisfree survival was 15.5 months, while median locoregional failure-free interval could not be calculated because survival rates were over $50 \%$ during the observation period (Fig. 3c and d), suggesting a high contribution of distant metastasis to recurrence. To elucidate factors relating to prognosis before treatment, we analyzed pre-NACIMRT factors affecting survivals and identified serum CA19-9 $>400 \mathrm{U} / \mathrm{ml}$ as a factor independently associated with overall survival (Table 4).

\section{Discussion}

$\mathrm{BRPC}$ is rare compared to resectable or unresectable PC, following difficulty in analyzing large series. Nagakawa et al. recently presented a large, retrospective study of BRPC with propensity-matched analyses to elucidate the effects of neoadjuvant radiation in 272 patients, revealing the $\mathrm{R} 0$ rate to be $87.2 \%$ in the neoadjuvant radiotherapy 


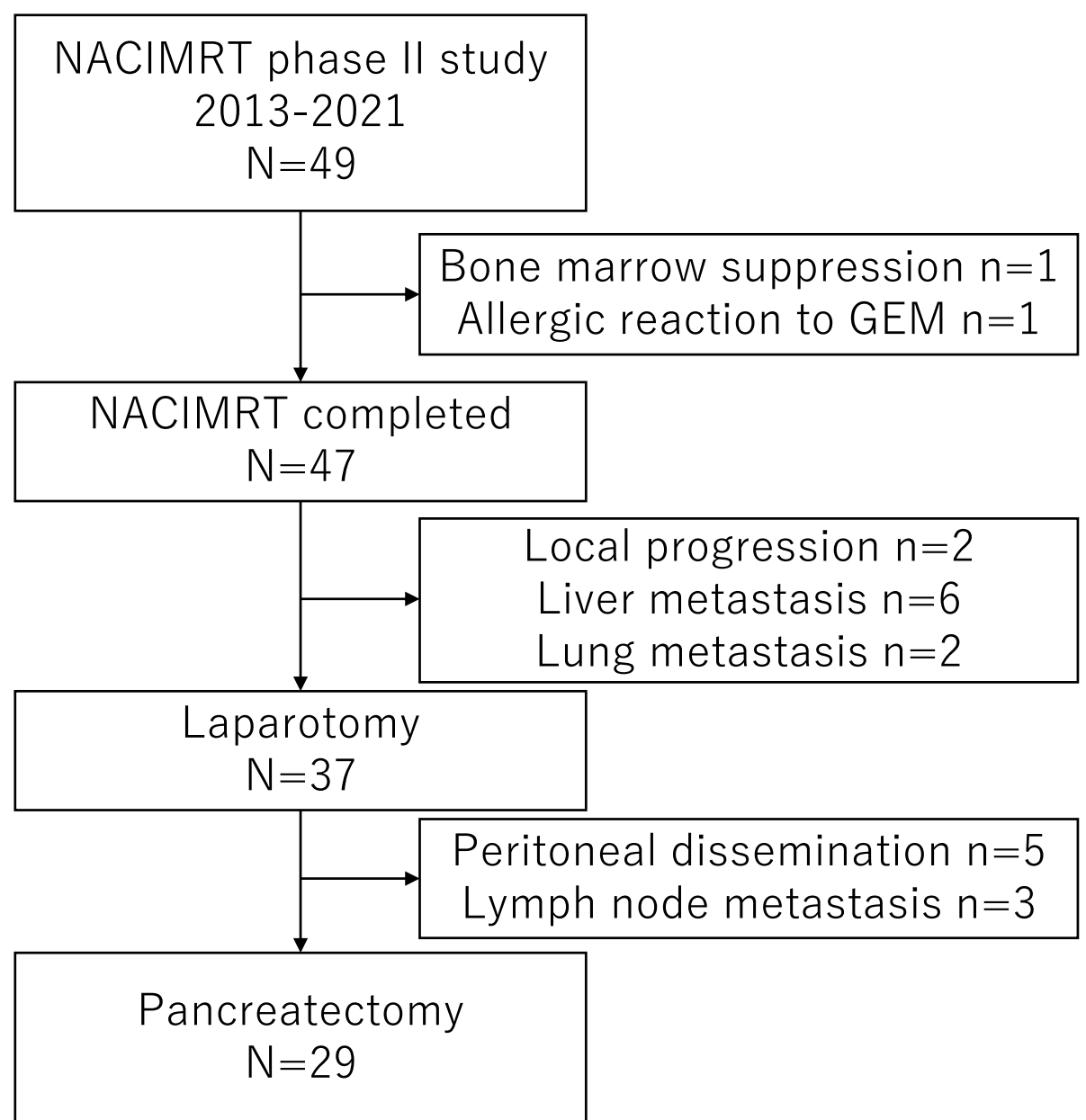

Fig. 2 Flow diagram of a phase 2 study with neoadjuvant IMRT. Forty-seven patients (95.9\%) completed NACIMRT with gemcitabine and 55.1\% (29/49) of the patients underwent pancreatectomy

group [19]. However, total resectability was unclear because of the retrospective design.

The current phase 2 prospective study investigated the safety and the efficacy of IMRT with concurrent gemcitabine in 49 BRPC-A patients. In this cohort, 47 out of 49 patients completed NACIMRT, there was no Grade 4 or 5 adverse events observed. Although there were 8 patients who suffered bile duct infection, all of these patients recovered with antibiotics. In terms of efficacy, R0 was completed in $93 \%$ of resected patients (27/29), and 55.1\% of initial BRPC-A patients (27/49) underwent curative resection. Our previous phase 2 study using gemcitabine and $\mathrm{S} 1$ as a neoadjuvant therapy for BRPC-A patients (NACGS study) showed that $73 \%$ of $\mathrm{R} 0$ resections and $60.8 \%$ of initial BRPC-A patients completed curative pancreatectomy, broadly comparable to the current study. However, for OS and PFS, our current NACIMRT study showed favorable survival compared to our previous NACGS study (NACIMRT vs. NACGS; median OS:
35.1 months vs. 21.7 months, median PFS: 15.5 months vs. 13.9 months) [8].

In comparison to other prospective trials, the PREOPANC study, a randomized phase 3 study for Resectable (R) and Borderline Resectable (BR) pancreatic cancer with neoadjuvant therapy, showed a good R0 rate (NAC $79 \%$ versus upfront $13 \%$ ) but low resection rate (NAC $52 \%$ versus upfront $64 \%$ ) in the subclass analysis with BRPC $(n=113)$, compared to immediate surgery [6]. Jang et al. compared NACRT and NAC in a BRPC with Randomized Control Trial (RCT) study $(n=50)$ and also found a high R0 rate with NACRT (82.4\%) compared to NAC (33.3\%), while resection was performed in $62.9 \%$ $(17 / 27)$ of NACRT patients, resulting in a $34 \%$ resection rate [7]. The recent JASPAC05 phase 2 trial found an R0 resection rate of $74 \%$ (29/39), while resection was performed in 55.7\% (29/52) [20] for BRPC. Although patients enrolled in the current study were limited to BRPC with artery abutment, our study also revealed a 
Table 2 Adverse Events ${ }^{*}$ Related to Neoadjuvant gemcitabine and Concurrent IMRT $(N=47)$

\begin{tabular}{llll}
\hline Adverse event & Grade 1-2, $\boldsymbol{n}(\%)$ & Grade 3, $\boldsymbol{n}$ (\%) & Grade 4-5, $\boldsymbol{n}$ (\%) \\
\hline White blood cell decreased & $42(86 \%)$ & $1(2 \%)$ & $0(0 \%)$ \\
Neutrophil count decreased & $28(57 \%)$ & $1(2 \%)$ & $0(0 \%)$ \\
Anemia & $38(76 \%)$ & $0(0 \%)$ & $0(0 \%)$ \\
Platelet count decreased & $30(61 \%)$ & $1(2 \%)$ & $0(0 \%)$ \\
Blood bilirubin increased & $14(29 \%)$ & $5(10 \%)$ & $0(0 \%)$ \\
AST increased & $22(45 \%)$ & $5(10 \%)$ & $0(0 \%)$ \\
ALT increased & $25(51 \%)$ & $6(12 \%)$ & $0(0 \%)$ \\
Hypoalbuminemia & $32(65 \%)$ & $1(2 \%)$ & $0(0 \%)$ \\
Serum AMY increased & $10(20 \%)$ & $4(8 \%)$ & $0(0 \%)$ \\
Allergic reaction & $3(6 \%)$ & $1(2 \%)$ & $0(0 \%)$ \\
Fatigue & $20(41 \%)$ & $0(0 \%)$ & - \\
Anorexia & $24(49 \%)$ & $0(0 \%)$ & $0(0 \%)$ \\
Diarrhea & $5(11 \%)$ & $0(0 \%)$ & $0(0 \%)$ \\
Mucositis/stomatitis & $2(4 \%)$ & $0(0 \%)$ & $0(0 \%)$ \\
Nausea & $22(45 \%)$ & $0(0 \%)$ & - \\
Vomiting & $8(16 \%)$ & $0(0 \%)$ & $0(0 \%)$ \\
Febrile neutropenia & - & $0(0 \%)$ & $0(0 \%)$ \\
Biliary tract infection & - & $8(16 \%)$ & $0(0 \%)$ \\
\hline
\end{tabular}

" Events were graded according to Common Terminology Criteria for Adverse Events (CTCAE) version 4.0. Abbreviations: IMRT intensity modulated radiotherapy, AST aspartate transaminase, $A L T$ alanine transaminase, $A L B$ albumin, $A M Y$ amylase

Table 3 Surgical outcomes and Pathological features

\begin{tabular}{ll}
\hline Surgical outcome & NACIMRT $(\boldsymbol{n}=\mathbf{2 9})$ \\
\hline Type of procedure (PD/DP/TP) & $24 / 4 / 1$ \\
Operation time (min) & $552(370-747)$ \\
Blood loss (ml) & $558(125-2900)$ \\
PV/SMV resection & $21(72.4 \%)$ \\
CHA/SMA resection & $4(13.8 \%)$ \\
CR-POPF (grade B or C) & $5(17.2 \%)$ \\
Clavian Dindo Illa $<$ & $4(13.7 \%)$ \\
re-operation & 0 \\
Duration of in-hospital stay & $26.5(15-58)$ \\
in-hospital death & 0 \\
Alb 1 month after resection (mg/dl) & $3.3(2.3-4.1)$ \\
ChE 1 month after resection (U/L) & $186(77-349)$ \\
relative dose intensity of adjuvant S1 (\%) & $75(0-100)^{\mathrm{a}}$ \\
Pathological features & \\
Tumor diameter (mm) & $22(0-55)$ \\
R0 & $27 / 29(93.1 \%)$ \\
positive LN metastasis & $10(34.4 \%)$ \\
Evans Grading (1/2a/2b/3/4) & $1 / 9 / 14 / 1 / 1$ \\
\hline
\end{tabular}

${ }^{a}$ not started in two patients (judged by a doctor) Abbreviations: $P D$ pancreaticoduodenectomy, $D P$ distal pancreatectomy, $T P$ total pancreatectomy, $P V$ portal vein, $S M V$ superior mesenteric vein, $C H A$ common hepatic artery, SMA superior mesenteric artery, CR-POPF clinically relevant postoperative pancreatic fistula, $A / b$ albumin, $C h E$ cholinesterase, $R O$ microscopically margin-negative resection, $L N$ lymph node high $\mathrm{R} 0$ rate with a fair resection rate. The low resection rate was mainly attributed to distant metastases, as 16 of 18 patients were found to have distant metastasis before pancreatectomy. In turn, this indicates preferable control of local progression by NACIMRT. Indeed, our locoregional failure-free intervals were significantly better than distant metastasis-free intervals after resection.

Despite the high R0 status and low resectability in the current study, as in previous studies, our current DFS and OS showed relatively high rates of long-term survivors compared to other NACRT studies for BR. Five-year survival rates were $28.2 \%$ for DFS and $38.3 \%$ for OS in the whole cohort. One important issue for this preferable outcome was that we applied IMRT, which can simultaneously reduce the dose to surrounding normal organs, while assuring adequate target dose coverage compared to conventional RT techniques. A recent study suggested that a customized clinical target volume that specifically includes the SMA and CA will improve coverage to this region and will account for individual and tumor variability [21]. The present series included the region around the SMA and CA in the CTV in addition to the GTV. In addition, we applied a hypofractionated dose of $42 \mathrm{~Gy}$ in 15 fractions, of which the biological dose is almost equivalent to the conventional standard treatment dose (48.6-50.4 Gy in fractions of 1.8 Gy). We did not intend to escalate the delivered dose because this study is a preoperative setting. The purpose of hypofractionated IMRT 


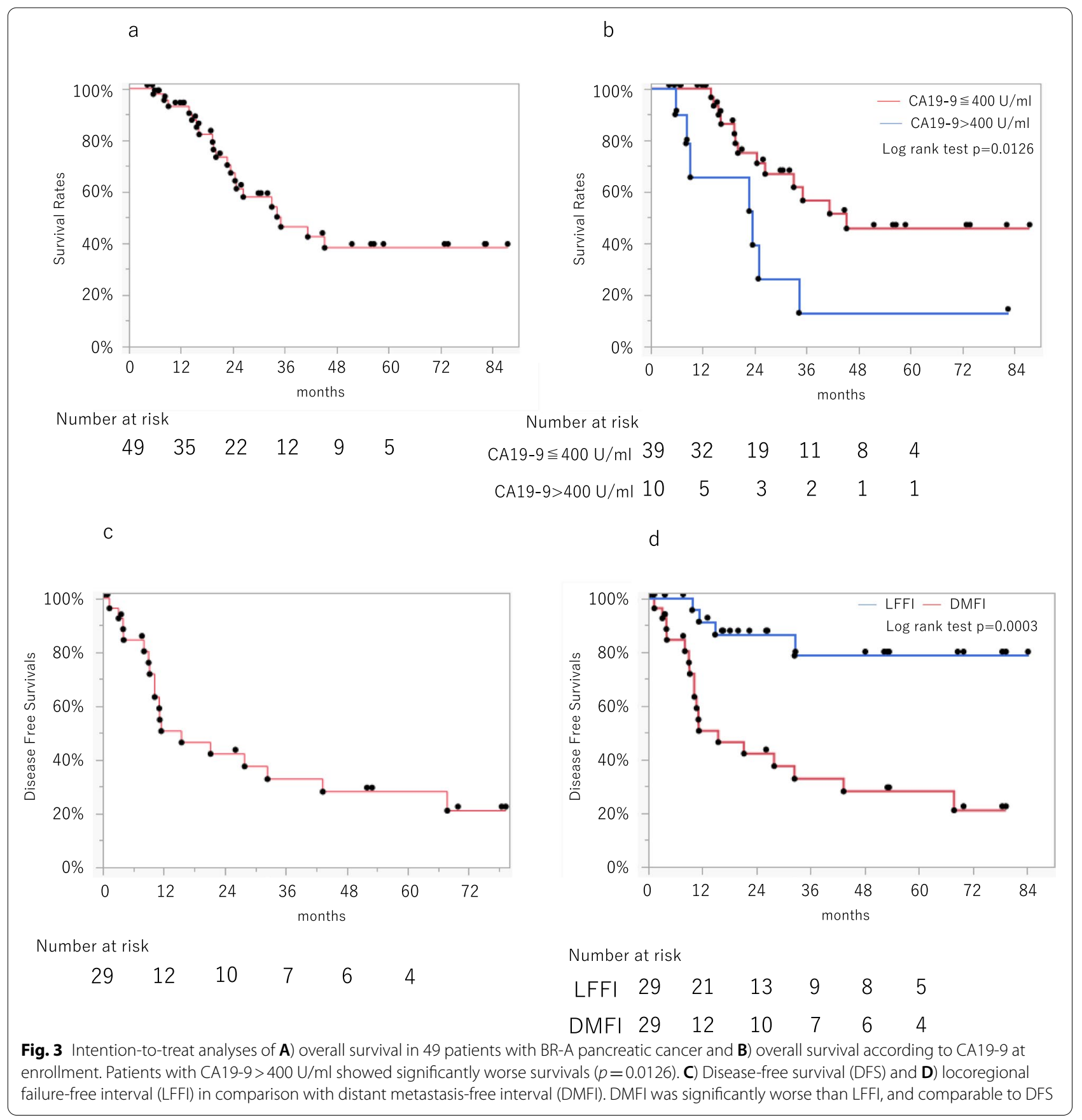

is to reduce the gastrointestinal toxicities with shortened radiation treatment period.

We have previously reported favorable outcomes of hypofractionated IMRT on Locally Advanced Pancreatic Cancer (LAPC) in elderly patients without severe toxicities [22]. One possibility is that current hypofractionated IMRT contributed to a high rate of S-1 introduction $(93.1 \%, 27 / 29)$ and a high relative dose intensity of S1 (median, 75\%) for adjuvant treatment of BRPC in
post-NAC patients. Indeed, a high relative dose intensity of adjuvant S1 therapy in BRPC patients treated by IMRT was reported in comparison to patients treated by standard CRT [23]. In the JASPAC05 study, a neoadjuvant trial of S-1 combined with conventional CRT, a lower $82 \%(22 / 27)$ of patients than in the present study were reported to receive adjuvant therapy after surgery, but the protocol for adjuvant therapy was not defined [20]. This might be another reason for the favorable outcomes 
Table 4 Factors at Enrollment associated with Overall Survivals

\begin{tabular}{|c|c|c|c|c|c|c|}
\hline \multirow[t]{2}{*}{ Variables } & \multicolumn{3}{|c|}{ Univariate analysis } & \multicolumn{3}{|c|}{ Multivariate analysis } \\
\hline & Odds ratio & $95 \% \mathrm{Cl}$ & $p$ value & Odds ratio & $95 \% \mathrm{Cl}$ & $p$ value \\
\hline age (per 1 year) & 1.02 & $0.96-1.10$ & 0.590 & & & \\
\hline male (v.s. female) & 0.79 & $0.32-1.94$ & 0.608 & & & \\
\hline head (v.s.body and tail) & 0.75 & $0.30-1.83$ & 0.520 & & & \\
\hline CA19-9>400 U/ml at enrollment & 3.08 & $1.22-7.79$ & 0.018 & 2.52 & $1.05-6.63$ & 0.043 \\
\hline diameter $>30 \mathrm{~mm}$ at enrollment & 1.85 & $0.76-4.48$ & 0.175 & 1.78 & $0.72-4.41$ & 0.212 \\
\hline BRPV at enrollment (v.s. without BRPV) & 1.79 & $0.59-5.36$ & 0.301 & & & \\
\hline NL at enrollment (per ratio) & 1.32 & $0.79-1.13$ & 0.200 & 1.24 & $0.52-1.19$ & 0.297 \\
\hline
\end{tabular}

Abbreviations: CA19-9 carbohydrate antigen 19-9, BRPV borderline resectable pancreatic cancer with portal vein involvement, NL neutrophil to leukocyte ratio

in our study. We verified the daily patient position using CBCT, but since huge advances in the technology such as hybrid MRI-Linac have been recently introduced that can help to deliver treatments in breath-hold irradiation, more accurate radiation treatment will be possible in the near future. [24].

In the current analysis evaluating pretreatment factors associated with survival, initial serum CA19-9 $>400$ $\mathrm{U} / \mathrm{ml}$ was an independent risk factor for overall survival, irrespective of tumor size. A reduction in serum CA19-9 or normalized CA19-9 after NAC is reportedly associated with good prognosis $[25,26]$, but categorizing those patients for whom neoadjuvant treatment should be applied beforehand, in addition to anatomical status, is difficult. Recently, high serum CA19-9 has been proposed as a "biological BR" in pancreatic cancer which shows worse survivals [27] and some recommend neoadjuvant treatment for such patients [28]. At the very least, the current study revealed that higher serum CA19-9 remains a worse prognostic factor, even with NACIMRT. Considering the high recurrence rate in terms of distant metastasis in this cohort, other modalities such as strong systemic chemotherapy aiming for reduction of micrometastases should be examined in combination with IMRT.

This study has several limitations. First, this was a study at a single institution. Although IMRT potentially reduces the toxicities of RT to normal organs with adequate target dose coverage, the GTV and CTV should be planned according to individual tumor location, spread and vascular anatomies in a standardized procedure. Standardizing the outcomes of IMRT in a multi-institutional study is thus difficult in terms of variety of quality. Second, the current enrolled BRPC patients were limited to patients with BRPC showing arterial abutment. Because a low R0 rate was most frequent in BRPC-A, we focused on this category of patients. The current study showed that local control was favorable even in BRPC-A, in which curative resection is more difficult and recurrence occurs more frequently at distant sites, suggesting that BRPC including BRPC-V and BRPC-A should be treated with the main focus on distant metastasis. Third, because this was not a randomized clinical trial, comparison with outcomes from other modalities such as FOLFIRINOX or gemcitabine/ nab paclitaxel is difficult.

\section{Conclusions}

The current GEM based NACIMRT phase 2 study showed a resectability rate of $55.1 \%$ for BRPC-A patients, who initially showed tumor abutment to major arteries. Median OS for the entire cohort was 35.1 months and the 5 -year OS rate was $38.3 \%$ with good feasibility of neoadjuvant IMRT with concurrent gemcitabine. As initial low CA19-9 is important for long survival with NACIMRT treatment, patients with high CA19-9 may need another strategy.

\section{Abbreviations}

BRPC: Borderline resectable pancreatic cancer; IMRT: Intensity-modulated radiation therapy; NACIMRT: Neoadjuvant chemotherapy using IMRT; BRPC-A: Borderline resectable pancreatic cancer with arterial abutment; R0: Microscopically margin-negative resection; CA19-9: Carbohydrate antigen 19-9; NCCN: The National Comprehensive Cancer Network; SMA: Superior mesenteric artery; CA: Celiac axis; CHA: Common hepatic artery; PHA: Proper hepatic artery; SMV: Superior mesenteric vein; PV: Portal vein; MDCT: Multidetectorrow computed tomography; FDG-PET: Positron emission tomography with 2-deoxy-2-[ $\left.{ }^{18} \mathrm{~F}\right]$ fluoro-D-glucose; MRI: Magnetic resonance imaging; EOB-MRI: MRI with contrast medium of gadolinium-ethoxybenzyl diethylenetriaminepentaacetic acid; GTV: Gross tumor volume; CTV: Clinical target volume; PTV: Planning target volume; LFFI: Locoregional failure-free interval; DMFI: Distant metastasis-free interval; DFS: Disease-free survival; OS: Overall survival; SUVmax: Maximum standardized uptake value; CEA: Carcinoembryonic antigen; NL ratio: Neutrophil-to-lymphocyte ratio; CR-POPF: Clinically relevant postoperative pancreatic fistula; Alb: Albumin; ChE: Cholinesterase; LAPC: Locally advanced pancreatic cancer.

\section{Acknowledgements}

The authors would like to thank all participants in our study. We would like to thank Forte Science Communications (www.forte-science.co.jp/) for English language editing. 


\section{Authors' contributions}

Study concept: TMa KN, TA, MM and KT. Study design: TMa, KN, TA, MM, MY, $\mathrm{NU}, \mathrm{AF}, \mathrm{SM}, \mathrm{MK}, \mathrm{HI}$, YK and KT. Acquisition of data: TMa, KN, TA, AS, YU, KN, YA, AK, NN, MM, SS, HK, TK and YK. Analysis and interpretation of data: TMa, KN and TA. Drafting the article: TMa, MY, and MK. Revising the draft critically for important intellectual content: KN, TA, AS, YU, KN, YA, AK, NN, MY, TMi, NU, AF, SM, MK, HI, MM, SU, and EH. Final approval of the version: TMa, KN, TA, AS, YU, KN, YA, AK, NN, MY, TMi, NU, AF, SM, MK, HI, MM, SS, KH, TK, YK, KT, SU, and EH. All authors read and approved the final manuscript.

\section{Funding}

This work was supported by JSPS KAKENHI Grant Number 21 K08732.

\section{Availability of data and materials}

The datasets used and/or analysed during the current study are available from the corresponding author on reasonable request.

\section{Declarations}

\section{Ethics approval and consent to participate}

The study protocol has been approved by the Ethics Committee of Kyoto University Graduate School and Faculty of Medicine (C0703) along with all necessary institutional and jurisdictional ethics committees. This study was performed in accordance with the ethical standards of the Declaration of Helsinki and its later amendments. Written informed consent for participation in this study was obtained from each participant.

\section{Consent for publication}

No identifiable data is presented.

\section{Competing interests}

The authors have no competing interests to declare regarding this study.

\section{Author details}

1Department of Surgery, Graduate School of Medicine, Kyoto University, Kyoto, Japan. ${ }^{2}$ Department of Radiation Oncology and Image-Applied Therapy, Graduate School of Medicine, Kyoto University, Kyoto, Japan. ${ }^{3}$ Department of Gastroenterology and Hepatology, Kyoto University, Kyoto, Japan. ${ }^{4}$ Department of Real World Data Research and Development, Graduate School of Medicine, Kyoto University, Kyoto, Japan. ${ }^{5}$ Department of Clinical Oncology, Kyoto University, Kyoto, Japan. ${ }^{6}$ Department of Diagnostic Imaging and Nuclear Medicine, Kyoto University, Kyoto, Japan.

Received: 28 October 2021 Accepted: 27 January 2022

Published online: 29 January 2022

\section{References}

1. Siegel RL, Miller KD, Jemal A. Cancer statistics, 2019. CA: a cancer journal for clinicians. 2019;69(1):7-34.

2. The Dynamic Statistics of the Population in 2019. 2020; https://www. mhlw.go.jp/toukei/saikin/hw/jinkou/kakutei19/index.html. Accessed 26th June, 2021

3. NCCN. NCCN clinical practice guidelines in oncology (NCCN guidelines). 2021; https://www.nccn.org/professionals/physician_gls/pdf/pancreatic. pdf. Accessed June 26th, 2021

4. Bockhorn M, Uzunoglu FG, Adham M, et al. Borderline resectable pancreatic cancer: a consensus statement by the International Study Group of Pancreatic Surgery (ISGPS). Surgery. 2014;155(6):977-88.

5. Ferrone CR, Marchegiani G, Hong TS, et al. Radiological and surgical implications of neoadjuvant treatment with FOLFIRINOX for locally advanced and borderline resectable pancreatic cancer. Ann Surg. 2015;261(1):12-7.

6. Versteijne E, Suker M, Groothuis K, et al. Preoperative Chemoradiotherapy Versus Immediate Surgery for Resectable and Borderline Resectable Pancreatic Cancer: Results of the Dutch Randomized Phase III PREOPANC Trial. Journal of clinical oncology : official journal of the American Society of Clinical Oncology. 2020;38(16):1763-73.

7. Jang JY, Han Y, Lee H, et al. Oncological Benefits of Neoadjuvant Chemoradiation With Gemcitabine Versus Upfront Surgery in Patients With
Borderline Resectable Pancreatic Cancer: A Prospective, Randomized, Open-label, Multicenter Phase 2/3 Trial. Ann Surg. 2018;268(2):215-22.

8. Masui T, Doi R, Kawaguchi Y, et al. Concurrent gemcitabine+S-1 neoadjuvant chemotherapy contributes to the improved survival of patients with small borderline-resectable pancreatic cancer tumors. Surgery today. 2016:46(11):1282-9.

9. Bittner MI, Grosu AL, Brunner TB. Comparison of toxicity after IMRT and 3D-conformal radiotherapy for patients with pancreatic cancer - a systematic review. Radiotherapy and oncology : journal of the European Society for Therapeutic Radiology and Oncology. 2015;114(1):117-21.

10. Lee KJ, Yoon HI, Chung MJ, et al. A Comparison of Gastrointestinal Toxicities between Intensity-Modulated Radiotherapy and ThreeDimensional Conformal Radiotherapy for Pancreatic Cancer. Gut and liver 2016:10(2):303-9.

11. Nakamura S, Murakami N, Inaba K, et al. After low and high dose-rate interstitial brachytherapy followed by IMRT radiotherapy for intermediate and high risk prostate cancer. BMC cancer. 2016;16:296

12. Merino T, San Francisco IF, Rojas PA, Bettoli P, Zuniga A, Besa P. Intensitymodulated radiotherapy versus radical prostatectomy in patients with localized prostate cancer: long-term follow-up. BMC cancer. 2013;13:530.

13. Liu F, Luo T, Jin T, et al. Advantages of using reduced-volume intensity modulated radiation therapy for the treatment of nasopharyngeal carcinoma: a retrospective paired study. BMC cancer. 2019;19(1):554.

14. Lin S, Lu JJ, Han L, Chen Q, Pan J. Sequential chemotherapy and intensitymodulated radiation therapy in the management of locoregionally advanced nasopharyngeal carcinoma: experience of 370 consecutive cases. BMC cancer. 2010;10:39

15. Nagakawa $Y$, Hosokawa Y, Nakayama H, et al. A phase II trial of neoadjuvant chemoradiotherapy with intensity-modulated radiotherapy combined with gemcitabine and S-1 for borderline-resectable pancreatic cancer with arterial involvement. Cancer Chemother Pharmacol. 2017;79(5):951-7.

16. Tran NH, Sahai V, Griffith KA, et al. Phase 2 Trial of Neoadjuvant FOLFIRINOX and Intensity Modulated Radiation Therapy Concurrent With Fixed-Dose Rate-Gemcitabine in Patients With Borderline Resectable Pancreatic Cancer. Int J Radiat Oncol Biol Phys. 2020;106(1):124-33.

17. Mori S, Hara R, Yanagi T, et al. Four-dimensional measurement of intrafractional respiratory motion of pancreatic tumors using a 256 multi-slice CT scanner. Radiotherapy and oncology : journal of the European Society for Therapeutic Radiology and Oncology. 2009;92(2):231-7.

18. Goto Y, Nakamura A, Ashida R, et al. Clinical evaluation of intensity-modulated radiotherapy for locally advanced pancreatic cancer. Radiation oncology. 2018;13(1):118.

19. Nagakawa Y, Sahara Y, Hosokawa Y, et al. Clinical Impact of Neoadjuvant Chemotherapy and Chemoradiotherapy in Borderline Resectable Pancreatic Cancer: Analysis of 884 Patients at Facilities Specializing in Pancreatic Surgery. Ann Surg Oncol. 2019;26(6):1629-36.

20. Takahashi S, Ohno I, Ikeda M, et al. Neoadjuvant S-1 With Concurrent Radiotherapy Followed by Surgery for Borderline Resectable Pancreatic Cancer: A Phase II Open-Label Multicenter Prospective Trial (JASPAC05). Annals of surgery. Oct 152020.

21. Bluemel TS, Kharofa J. Comparison of Radiation Treatment Volumes for Borderline Resectable Pancreatic Cancer in Contemporary Clinical Trials. Am J Clin Oncol. 2020;43(9):648-53.

22. Iwai T, Yoshimura M, Ashida R, et al. Hypofractionated intensity-modulated radiotherapy with concurrent chemotherapy for elderly patients with locally advanced pancreatic carcinoma. Radiation oncology. 2020;15(1):264

23. Masui T, Takaori K, Anazawa T, et al. A Prospective Study of Intensitymodified Radiation Therapy in Comparison with Conventional 3D-RT for BR Pancreatic Cancer Patients with Arterial Involvement. Anticancer Res. 2017:37(12):7023-30.

24. Massaccesi M, Cusumano D, Boldrini L, et al. A new frontier of image guidance: Organs at risk avoidance with MRI-guided respiratory-gated intensity modulated radiotherapy: Technical note and report of a case. J Appl Clin Med Phys. 2019;20(6):194-8.

25. Rose JB, Edwards AM, Rocha FG, et al. Sustained Carbohydrate Antigen 19-9 Response to Neoadjuvant Chemotherapy in Borderline Resectable Pancreatic Cancer Predicts Progression and Survival. Oncologist. 2020;25(10):859-66. 
26. Aoki S, Motoi F, Murakami Y, et al. Decreased serum carbohydrate antigen 19-9 levels after neoadjuvant therapy predict a better prognosis for patients with pancreatic adenocarcinoma: a multicenter case-control study of 240 patients. BMC cancer. 2019;19(1):252.

27. Deng GC, Lv Y, Yan H, et al. Nomogram to predict survival of patients with advanced and metastatic pancreatic Cancer. BMC cancer. 2021;21(1):1227.

28. Takahashi H, Yamada D, Asukai K, et al. Clinical implications of the serum CA19-9 level in "biological borderline resectability" and "biological downstaging" in the setting of preoperative chemoradiation therapy for pancreatic cancer. Pancreatology : official journal of the International Association of Pancreatology. 2020;20(5):919-28.

\section{Publisher's Note}

Springer Nature remains neutral with regard to jurisdictional claims in published maps and institutional affiliations.

- fast, convenient online submission

- thorough peer review by experienced researchers in your field

- rapid publication on acceptance

- support for research data, including large and complex data types

- gold Open Access which fosters wider collaboration and increased citations

- maximum visibility for your research: over $100 \mathrm{M}$ website views per year

At BMC, research is always in progress.

Learn more biomedcentral.com/submissions 